\title{
Effect of $\mathrm{CoQ}_{10}$ Supplementation on Oxidative Stress and Muscle Bioenergetics in Type II Diabetes: A Pilot Study
}

\author{
Joohee Sanders, $\mathrm{PhD}^{1^{*}}$, Sinclair Smith, $P h D^{2}$ and Carsten Sanders, PhD \\ ${ }^{1}$ Department of Exercise Science, Shippensburg University, Shippensburg, PA, USA \\ ${ }^{2}$ College of Nursing and Health Professions, Drexel University, Philadelphia, PA, USA \\ ${ }^{3}$ Department of Physical Sciences, Kutztown University, Kutztown, PA, USA \\ *Corresponding author: Joohee Im Sanders, PhD, Department of Exercise Science, Shippensburg University, 1871 Old \\ Main Drive, Shippensburg, PA 17257, USA, Tel: 717-477-1378, Fax: 717-477-4083
}

\begin{abstract}
Objectives: To compare oxidative stress, mitochondrial oxidative phosphorylation capacity, microvascular function, and exercise performance of type II diabetics (T2D) to that of control subjects and to determine whether Co-enzyme $Q_{10}\left(C_{o} Q_{10}\right)$ supplementation has any effects on these measures.

Methods: Fourteen older adults (7 T2D patients with mild peripheral arterial disease (PAD) $(69.9 \pm 5.1$ years) and 7 age-matched controls (67.0 \pm 8.9 years) participated in the study. Each participant went through 2 week placebo followed by 2 week $\mathrm{CoQ}_{10}$ supplementation trials. During each trial, participants performed plantar flexion exercise during which gastrocnemius phosphocreatine $(\mathrm{PCr}), \mathrm{pH}$, oxygen $\left(\mathrm{O}_{2}\right)$ saturation and BFindex were measured using ${ }^{31} \mathrm{P}$ magnetic resonance spectroscopy (MRS) and near infrared spectroscopy (NIRS). Results: Comparing T2D to controls, significant differences were observed in $\mathrm{PCr}_{\text {re }}(22.3 \pm 13.4$ vs. $\left.49.4 \pm 36.6 \mathrm{mM} \cdot \mathrm{kg}^{-1} \cdot \mathrm{min}^{-1}\right)$ and BFindex $(5.3 \pm 2.9$ vs. $\left.9.7 \pm 2.9 \% \cdot \mathrm{s}^{-1}\right)(p=0.03)$. Moreover, $\mathrm{O}_{2}$ saturation recovery was significantly longer for T2D (70.9 \pm 52.1 vs. $31.8 \pm 8.4$ $\mathrm{s} ; p<0.01)$. When comparing placebo trial to $\mathrm{CoQ}_{10}$ trial, significant reduction in Malondialdehyde (MDA), after $\mathrm{CoQ}_{10}$ trial, was observed in controls $(1.29 \pm 0.41$ vs. $0.88 \pm 0.35$ $\mathrm{uM}, p<0.05)$ but not in T2D $(1.31 \pm 0.19$ vs. $1.47 \pm 0.36 \mathrm{uM}$, $p>0.05$ ). With the $\mathrm{CoQ}_{10}$ trial, a trend of improved $\mathrm{PCr} / \mathrm{Pi}$ in $\mathrm{T} 2 \mathrm{D}$ patients at rest was seen $(6.87 \pm 1.2$ to $8.59 \pm 2.7)$. However, no other significant changes were observed with $\mathrm{CoQ}_{10}$ supplementation for either group, including $\mathrm{pH}, \mathrm{PCr}$ recovery (T2D: $22.3 \pm 13.4$ vs. $23.2 \pm 11.4 \mathrm{mM} \cdot \mathrm{kg}^{-1} \cdot \mathrm{min}^{-1}$; $\mathrm{CON}: 49.4 \pm 36.6$ vs. $43.7 \pm 33.3 \mathrm{mM} \cdot \mathrm{kg}^{-1} \cdot \mathrm{min}^{-1}, p=0.9$ ), and BFindex (T2D: $5.3 \pm 2.9$ vs. $3.9 \pm 2.2 \% \cdot \mathrm{s}^{-1}$; CON: $9.7 \pm$ 2.9 vs. $\left.9.2 \pm 2.4 \% \cdot \mathrm{s}^{-1}, p=0.5\right)$.
\end{abstract}

Conclusion: Noted reduction in MDA seen in healthy, older individuals suggests a positive antioxidant effect of $\mathrm{CoQ}_{10}$ supplementation. However, when PAD is present, as can be the case of T2D, two weeks of $\mathrm{CoQ}_{10}$ supplementation at $200 \mathrm{mg} \cdot$ day $^{-1}$ may not be long enough or potent enough to produce similar effects.

\section{Keywords}

$\mathrm{CoQ}_{10}$, Diabetes, Antioxidant, Metabolic function, Hemodynamics, Exercise

\begin{abstract}
Abbreviations
$\triangle \mathrm{BV}$ : Blood Volume Change; $\mathrm{ABI}$ : Ankle Brachial Index; AOX: Antioxidant; ATP: Adenosine Triphosphate; BFindex: Microvascular blood flow; BMI: Body Mass Index; CoQ10: Coenzyme Q10; ETC: Electron Transport Chain; H+: Proton; $\mathrm{Hb} / \mathrm{Mb}-\mathrm{oxy}$ : Hemoglobin/Myoglobin- $\mathrm{O}_{2}$ Saturation; ipH: Intracellular pH; MDA: Malondialdehyde; NIRS: Near Infrared Spectroscopy; NO: Nitric Oxide; NOS: Nitric Oxide Synthase; MRS: Magnetic Resonance Spectroscopy; PAD: Peripheral Arterial Disease; PCr: Phosphocreatine; PCrre: PCr Resynthesis Rate; RM: Repetition Maximum; ROS: Reactive Oxygen Species; SSI: Steady State Incremental Exercise; T2D: Type 2 Diabetes; T50NIR: Half-time to Recovery; VO: Venous Occlusion
\end{abstract}

\section{Introduction}

In the United States, 30.3 million people (9.4\% of the population) have diabetes, most of whom suffer from type 2 diabetes (T2D) [1]. Hyperglycemia in T2D may alter glucose metabolic pathways leading to formation of unfavorable by-products such as reactive oxygen species (ROS) [2]. Elevated ROS and its subsequent oxidati-

Citation: Sanders J, Smith S, Sanders C (2020) Effect of $\mathrm{CoQ}_{10}$ Supplementation on Oxidative Stress and Muscle Bioenergetics in Type II Diabetes: A Pilot Study. Int J Diabetes Clin Res 7:116. doi. org/10.23937/2377-3634/1410116

Accepted: January 28, 2020: Published: January 30, 2020

Copyright: (C) 2020 Sanders J, et al. This is an open-access article distributed under the terms of the Creative Commons Attribution License, which permits unrestricted use, distribution, and reproduction in any medium, provided the original author and source are credited. 
ve stress, due to hyperglycemia in T2D, have been proposed as a major contributing factor leading to diabetic complications [3-5] including vascular dysfunction [6-8]. Peripheral Arterial Disease (PAD) is a common finding in T2D [9] in which blood flow and subsequent $\mathrm{O}_{2}$ supply to working muscles are impaired thereby limiting functional capacity $[10,11]$. Moreover, T2D associated with $P A D$, has been shown to increase mortality rate in this population [12].

Another factor contributing to impaired functional capacity in T2D is mitochondrial dysfunction. Studies have reported decreased oxidative phosphorylation activity $[13,14]$, as well as mitochondrial DNA damage [15], in T2D. Moreover, defects in the capacity to metabolize glucose and fatty acids results in accumulation of fatty acids and triacylglycerol in non-adipose tissues, including skeletal muscle [16]. These accumulating fatty acids are prone to lipid peroxidation increasing ROS, a process thought to be one of the major causes of mitochondrial damage [17].

While hyperglycemia in T2D increase ROS production, it can also lead to glycation of antioxidant (AOX) enzymes thereby reducing AOX availability $[18,19]$. Thus, hyperglycemia upregulates ROS formation while down-regulating AOX formation leading to an even higher level of oxidative stress. For example, mitochondrial reducing equivalents, such as co-enzyme $\mathrm{Q}_{10}\left(\mathrm{CoQ}_{10}\right)$, have been found to be deficient in T2D $[20,21]$. This suggests that a quantitative and/or functional deficiency in $\mathrm{CoQ}_{10}$ may potentially occur in T2D, further diminishing metabolic efficiency and functional capacity.

$\mathrm{CoQ}_{10}$ is a potent antioxidant $[22,23]$ and a component of the ETC which has been shown to improve mitochondrial $[24,25]$ and vascular function $[26,27]$. Furthermore, $\mathrm{CoQ}_{10}$ has shown to reduce fasting plasma glucose in diabetes [23]. Thus, supplemental $\mathrm{CoO}_{10}$ has the potential to improve functional capacity of T2D and possibly reduce the progression of disease. In the present study, we examined and compared these parameters of T2D patients to that of control subjects and how short-term dietary $\mathrm{CoQ}_{10}$ supplementation may alter these measures. We hypothesized that; 1) T2D patients would exhibit impaired mitochondrial and microvascular functions, and exercise performance when compared to age-matched control subjects and 2) T2D patients would show significant improvements in these measures after $\mathrm{CoQ}_{10}$ supplementation.

\section{Methods}

\section{Subjects}

Fourteen older individuals were recruited to participate in the study (7 T2D patients with a mild case of PAD (Fontain class lla) (Age: $69.9 \pm 5.1 \mathrm{yrs}$ ) and 7 age, gender and activity matched non-diabetic individuals (Age: $67.0 \pm 8.9 \mathrm{yrs}$ ). All subjects were non-smokers and en- gaged in no more than one planned physical activity per week. All T2D patients were previously diagnosed with T2D (fasting glucose $\geq 126 \mathrm{mg} / \mathrm{dL}$ ) and in good glycemic control. The study protocol was approved by the Institutional Review Board at the University of Pennsylvania (IRB\#: 803931). Informed consent was obtained from each subject before enrollment of the study.

Prior to the study, each subject's laboratory and physical examination information, including vital signs, height, weight, diet, activity habits, medication, ankle brachial index ( $\mathrm{ABI}$ ), and blood work were obtained. Once enrolled in the study, the same measures and questionnaire were obtained during each visit to monitor and record any changes during the course of the study. All study subjects were encouraged to stay on the same diet, medication, and activity level throughout the study.

\section{Experimental procedure}

This study used a non-randomized, single blinded, cross-over design, where all T2D patients and control subjects received 2 weeks of placebo treatment first, followed by 2 weeks of $\mathrm{CoQ}_{10}$ treatment at $200 \mathrm{mg} \cdot$ day $^{-1}$ (100 mg capsule, twice a day). The treatment order was not randomized due to a long wash out period of $\mathrm{CoQ}_{10}$. An oral, over-the-counter $\mathrm{CoQ}_{10}$ dietary supplement was used (Swanson Health Products, Inc., Fargo, ND). To account for a possible order effect, an initial baseline trial was conducted before the placebo trial.

Each subject was asked to report three times; 1) Baseline (visit 1); 2) 2 weeks post placebo (visit 2); and 3) 2 weeks post $\mathrm{CoQ}_{10}$ supplement (visit 3). Identical exercise testing was performed during each visit. Blood samples were drawn from the antecubital vein before and after exercise in all trials to measure changes in Malondialdehyde (MDA) level to estimate oxidative stress status.

\section{Exercise protocol}

All exercise protocols were performed in a 2 Tesla, $75 \mathrm{~cm}$ bore MRS magnet. Co-registration of ${ }^{31} \mathrm{P}-\mathrm{MRS}$ and NIRS (Cogniscope; NIM, Inc.) was performed to observe dynamic changes of phosphate $\left({ }^{31} \mathrm{P}\right)$ metabolites, intracellular $\mathrm{pH}(\mathrm{ipH})$ and total hemoglobin/myoglobin $-\mathrm{O}_{2}$ saturation ( $\mathrm{Hb} / \mathrm{Mb}$-oxy) in the medial gastrocnemius at rest, during and after exercise. For exercise protocol, dynamic plantar flexion in supine position was performed, using workloads relative to pre-determined one repetition maximum (RM) (Figure 1). For T2D group, the leg that the patient was having the most pain was used throughout the study.

12 s Dynamic exercise: After obtaining resting measures, each subject performed a 12-s dynamic plantar flexion exercise ( 2 contraction/s) at $40 \%$ of $1 \mathrm{RM}$. All subjects stopped exercising after 12 -s or when medial gastrocnemius muscle $\mathrm{PCr}$ level declined to $50 \%$ of the resting $\mathrm{PCr}$, whichever came first [28]. From the MRS 


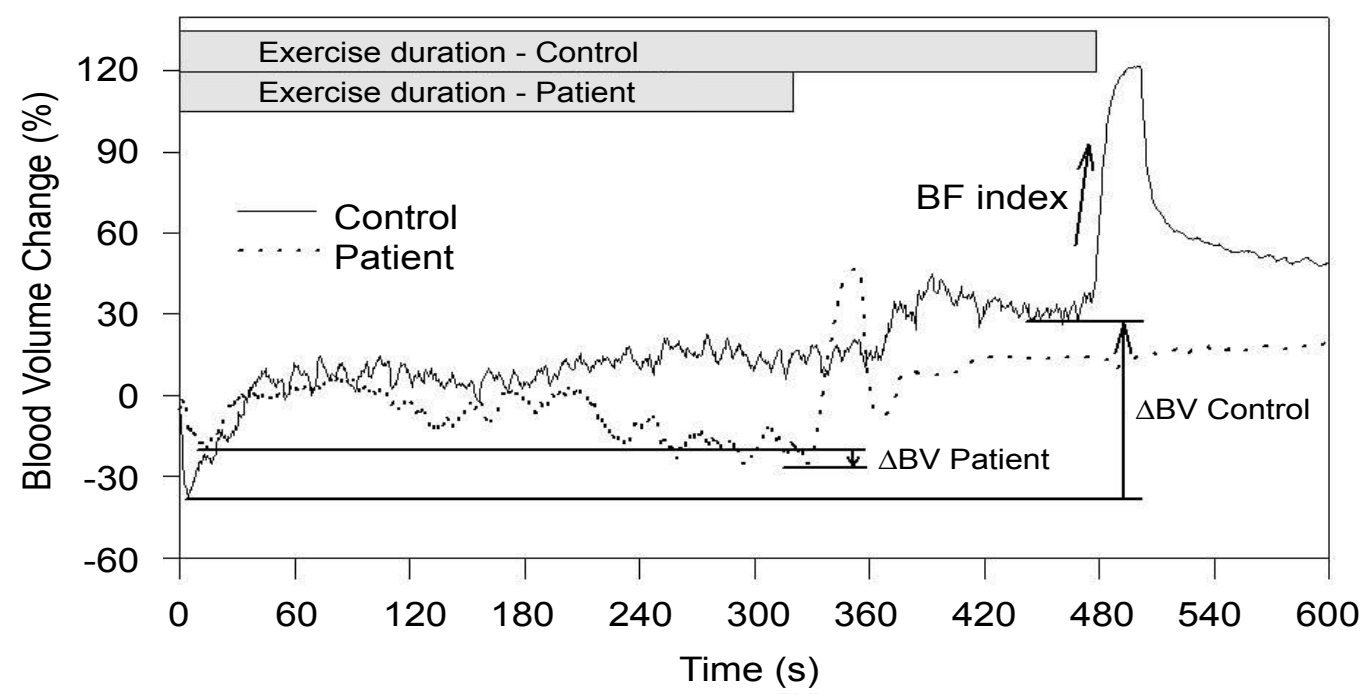

Figure 1: Typical changes in blood volume during SSI exercise in a control subject (solid line) and a T2D patient (dotted line).

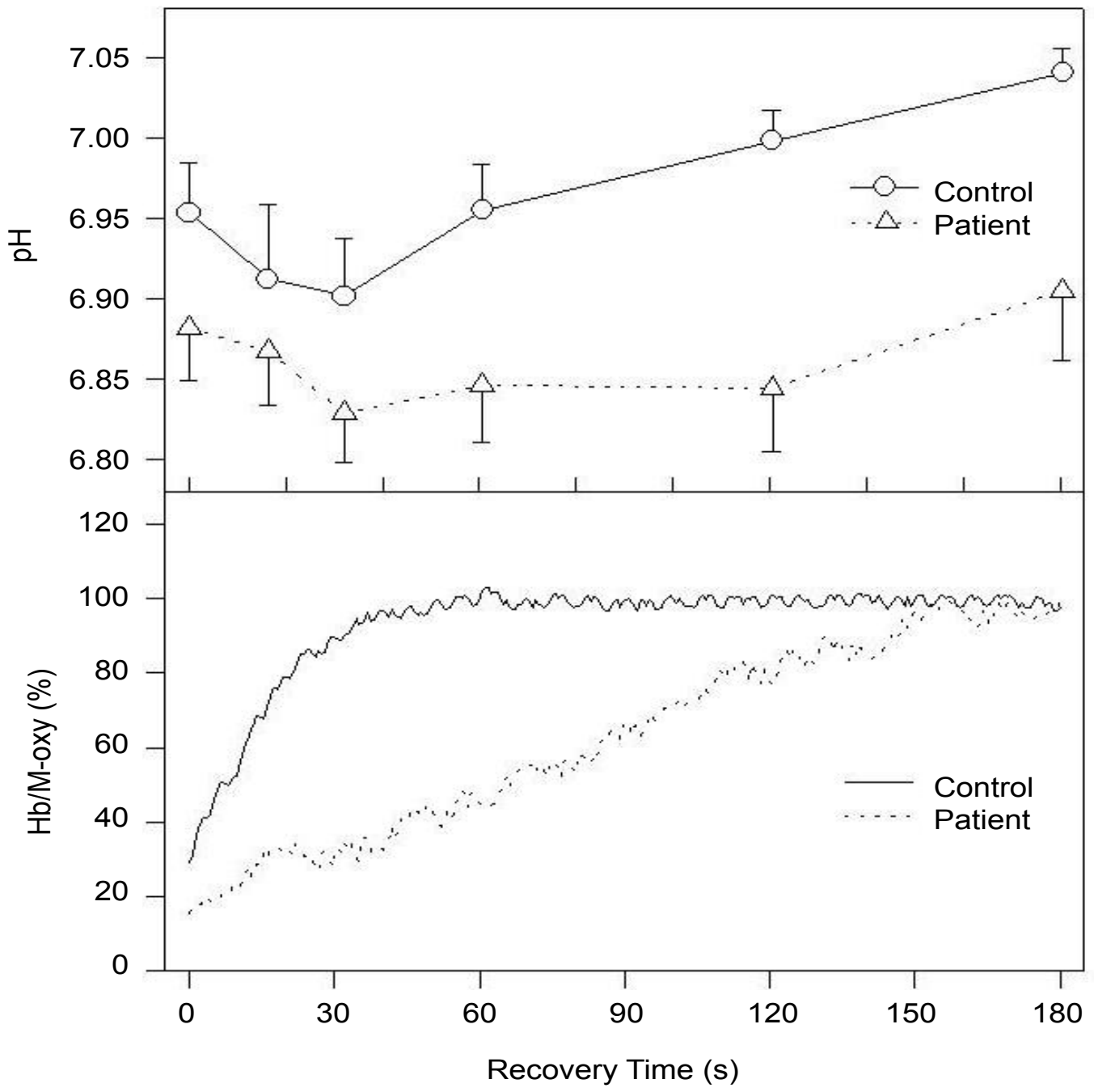

Figure 2: Typical changes in ipH (circle and triangle; top panel) and $\mathrm{Hb} / \mathrm{Mb}-\mathrm{oxy}$ (solid and dotted lines; bottom panel) after SSI exercise in a control subject (circle and solid line) and a T2D patient (triangle and dotted line).

spectra, post-exercise $\mathrm{PCr}$, ipH, and $\mathrm{PCr}$ re-synthesis rate $\left(\mathrm{PCr}_{\text {re }}\right)$ were measured as a function of mitochondrial oxidative phosphorylation capacity. From NIR spectra, $\mathrm{Hb} / \mathrm{Mb}-\mathrm{Oxy}$ during exercise was measured as a function of $\mathrm{O}_{2}$ utilization. Half-time to recovery $\left(\mathrm{T}_{50 \mathrm{NIR}}\right)$, which is the time from cessation of exercise to $50 \%$ of resting $\mathrm{Hb} / \mathrm{Mb}$-oxy, was measured as a function of $\mathrm{O}_{2}$ delivery. Recovery delay was measured from cessation of exercise to the start of $\mathrm{Hb} / \mathrm{Mb}$-oxy recovery.

Steady State Incremental Exercise (SSI): Each 
subject also performed SSI by pushing down the pedal against a set resistance at a rate of 30 plantar flexions/min with a 1-s work/rest cycle (Figure 2). Starting workload was set at $30 \%$ of 1 RM and increased by $10 \%$ every two minutes. Exercise was stopped when $\mathrm{PCr}$ level declined to $<50 \%$ of the resting PCr. Exercise capacity was determined by the time it took for each subject to deplete $50 \%$ of resting PCr. From NIR spectra, blood volume change $(\triangle B V)$ during exercise and initial rate of total $\mathrm{Hb}$ change $\left(\Delta \mathrm{tHB}_{\text {rate }}\right)$ immediately after exercise were used to determine BFindex. From MRS spectra, post-exercise $\mathrm{PCr}$, ipH, and ipH recovery were measured.

\section{Data analysis}

All data were analyzed using a commercially available statistical software tool pack (Sigma Stat). A twoway ANOVA (Analysis of Variance) with placebo vs. $\mathrm{CoQ}_{10}$ trials and T2D vs. control subjects as factors were conducted on all variables. Significance level $(\alpha)$ was set to 0.05 . To examine relationships between MRS and NIRS metabolic measures, a pearson product-moment correlation was used.

\section{Results}

\section{T2D patients vs. Control subjects}

Resting response: $\mathrm{T} 2 \mathrm{D}$ and control subjects' resting variables are summarized in Table 1 . There were no significant differences in anthropometric, heart rate (HR) or blood pressure (BP) measures. T2D patients' $\mathrm{ABI}$ for both right and left were below 1 (range $=0.61 \sim 0.95$ ) and were significantly lower than the control subjects $(p=0.001)$. T2D patients' resting ${ }^{31} \mathrm{P}$ spectra revealed significantly smaller $\mathrm{PCr}(p=0.04), \mathrm{PCr} / \mathrm{Pi}(p=0.01)$, and larger $\mathrm{Pi}(p=0.04)$ (Table 1$)$.

Exercise response: End exercise $\mathrm{PCr}(p=0.745)$, $\mathrm{PCr}$ depletion rate at the onset of exercise and end exercise ipH were not significantly different between T2D and control subjects for either $12 \mathrm{~s}(p=0.634)$ or $\mathrm{SSI}(p=0.165)$ exercise (Figure 3$)$. ipH remained above 6.75 (range $=6.85$ to 6.96 ) for all groups during all exercise tests, indicating that exercises were performed under relatively aerobic conditions as designed. 12 -s end exercise $\mathrm{Hb} / \mathrm{Mb}$-oxy (\%) was not significantly different in T2D when compared to control subjects (Table 1 and Figure 3) $(p=0.547)$. During SSI exercise,

Table 1: Metabolic and hemodynamic responses at rest, during and after exercise in T2D patients and control subjects.

\begin{tabular}{|c|c|c|c|}
\hline & T2D (n= 8) & Control $(n=7)$ & $\boldsymbol{P}$ \\
\hline \multicolumn{4}{|l|}{ Resting } \\
\hline $\mathrm{PCr}(\mathrm{mM})$ & $37.87 \pm 3.24$ & $41.09 \pm 2.14$ & 0.04 \\
\hline $\mathrm{Pi}(\mathrm{mM})$ & $5.97 \pm 0.99$ & $4.78 \pm 1.06$ & 0.04 \\
\hline $\mathrm{PCr} / \mathrm{Pi}$ & $6.52 \pm 1.30$ & $8.93 \pm 1.81$ & 0.01 \\
\hline $\mathrm{Cr}(\mathrm{mM})$ & $5.68 \pm 0.49$ & $6.16 \pm 0.32$ & 0.04 \\
\hline ADP (uM) & $7.78 \pm 0.29$ & $7.67 \pm 0.72$ & 0.678 \\
\hline $\mathrm{pH}$ & $7.02 \pm 0.02$ & $7.02 \pm 0.05$ & 0.880 \\
\hline \multicolumn{4}{|l|}{ Exercise \& Recovery } \\
\hline SSI Ex. Duration (s) & $321.00 \pm 116.77$ & $420.00 \pm 105.48$ & 0.03 \\
\hline \multicolumn{4}{|l|}{ NIRS } \\
\hline End $\mathrm{Hb} / \mathrm{Mb}-\mathrm{oxy}(\%)$ & $24.88 \pm 6.91$ & $30.39 \pm 13.82$ & 0.547 \\
\hline $\mathrm{T}_{50}(\mathrm{~s})$ & $77.35 \pm 55.21$ & $30.80 \pm 8.60$ & 0.05 \\
\hline Recovery Delay (s) & $32.41 \pm 16.53$ & $14.18 \pm 7.02$ & 0.02 \\
\hline$\Delta \mathrm{BV}(\%)$ & $-5.12 \pm 55.87$ & $65.90 \pm 22.73$ & 0.008 \\
\hline BFindex (\%/s) & $4.98 \pm 2.17$ & $10.11 \pm 3.90$ & 0.01 \\
\hline \multicolumn{4}{|l|}{ MRS } \\
\hline End PCr (\%) & $46.31 \pm 16.81$ & $49.40 \pm 17.60$ & 0.745 \\
\hline $\mathrm{dPCr} / \mathrm{dt}(\% / \mathrm{s})$ & $-4.00 \pm 1.80$ & $-3.73 \pm 1.79$ & 0.786 \\
\hline $\mathrm{PCr}_{\mathrm{re}}(\mathrm{mM} / \mathrm{kg} / \mathrm{min})$ & $22.31 \pm 13.35$ & $49.37 \pm 36.62$ & 0.015 \\
\hline Tc (s) & $67.21 \pm 69.51$ & $35.59 \pm 23.65$ & 0.119 \\
\hline pH End ex. (12-s) & $6.979 \pm 0.047$ & $7.006 \pm 0.034$ & 0.634 \\
\hline pH End ex. (SS) & $6.893 \pm 0.084$ & $6.954 \pm 0.083$ & 0.165 \\
\hline pH 1 min post (12-s) & $6.910 \pm 0.043$ & $7.007 \pm 0.057$ & 0.002 \\
\hline pH 1 min post (ss) & $6.851 \pm 0.086$ & $6.955 \pm 0.076$ & 0.028 \\
\hline
\end{tabular}

Note: PCr: Phosphocreatine; Pi: Inorganic phosphate; PCr/Pi: Ratio of PCr to Pi concentration; ipH: intracellular $\mathrm{pH}$; $\mathrm{T}_{50 \mathrm{NIR}}$ : Time to $50 \%$ of Hemoglobin/Myoglobin-reoxygenation from cessation of exercise; $\triangle \mathrm{BV}$ : Blood volume change, BFindex: Blood flow index; End PCr: End exercise PCr; dPCr/dt: Change in $\mathrm{PCr}$ over change in time; $\mathrm{PCr}_{\mathrm{re}}$ : Phosphocreatine resynthesis rate; Tc: Time constant; 12-s: 12-s all out exercise; ss: steady state incremental exercise. Data are expressed in mean \pm SD. 


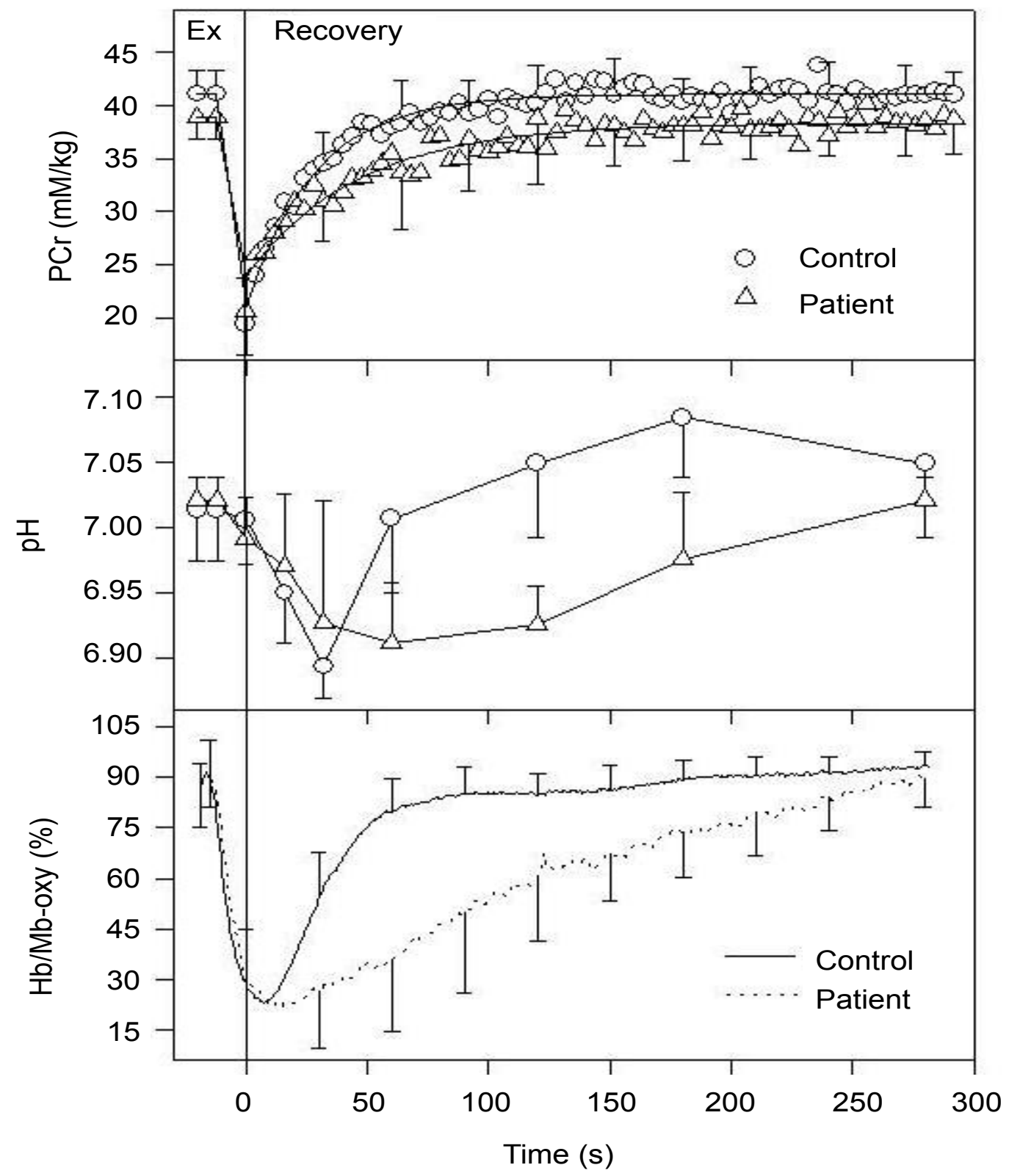

Figure 3: Changes in $\mathrm{PCr}$ (top panel), ipH (middle panel) and Hb/Mb-oxy (bottom panel) at rest, during and after $12 \mathrm{~s}$ exercise in control subjects (circle and solid line) and T2D patients (triangle and dotted line).

T2D reached $50 \%$ of resting $\mathrm{PCr}$ significantly earlier than control subjects resulting in shorter exercise duration ( $p=0.03$ ) (Table 1 ). A significantly larger increase of $\triangle B V$ was observed in control subjects whereas in $T 2 D$, after an initial increase, $\triangle B V$ either stayed the same or decreased ( $p=0.008$ ) (Figure 1 ).

Recovery response: $\mathrm{PCr}_{\text {re }}$ and Tc after 12-s exercise was significantly less in T2D when compared to control subjects $(p=0.02)$ (Figure 3 and Table 1). When $\mathrm{PCr}_{\text {re }}$ and Tc were correlated to NIR measures, strong and significant relationships were shown between $\mathrm{PCr}_{\text {re }}$ and $\mathrm{T}_{50 \mathrm{NIR}}(\mathrm{r}=-0.46 ; p=0.01), \mathrm{PCr}_{\text {re }}$ and recovery delay $(\mathrm{r}=$ -0.46; $p=0.01), \mathrm{PCr}_{\mathrm{re}}$ and $\Delta \mathrm{BV}(\mathrm{r}=0.60 ; p<0.001)$. ipH recovery was also significantly slower in T2D for both 12 -s $(p=0.002)$ and SSI $(p=0.03)$ exercises (Figure 2 and Figure 3 ). $\mathrm{T}_{\text {50NIR }}$ in $\mathrm{O}_{2}$ saturation was significantly slower in T2D when compared to control subjects ( $p=$ 0.05) (Figure 2, Figure 3 and Table 1). Moreover, there was a significant delay in $\mathrm{HbO}_{2}$ recovery in $\mathrm{T} 2 \mathrm{D}(p=0.02)$ (Table 1).

\section{Effect of $\mathrm{CoQ}_{10}$}

Resting response: A significant reduction in MDA, after $\mathrm{CoQ}_{10}$ trial, was observed in control subjects (1.29 \pm 0.41 vs. $0.88 \pm 0.35$ uM, $p<0.05$ ) but not in T2D (1.31 \pm 0.19 vs. $1.47 \pm 0.36 \mathrm{uM}, p>0.05$ ) (Figure 4). With $\mathrm{CoQ}_{10}$ supplementation, reduced $\mathrm{Pi}$ and elevated $\mathrm{PCr} / \mathrm{Pi}$ were seen in T2D to a point where significant differences seen, prior to $\mathrm{CoQ}_{10}$, between the groups were diminished. However, these changes were not statistically significant $(p>0.05)$ (Table 2).

Exercise response: Similar to resting responses, post exercise MDA was significantly lower, after $\mathrm{CoQ}_{10}$ trial, when compared to placebo trial in controls (1.17 \pm 0.28 vs. $1.51 \pm 0.17 \mathrm{uM}, p<0.05)$ but not in T2D (1.64 \pm 0.29 vs. $1.56 \pm 0.31 \mathrm{uM}, p>0.05)$ 
Table 2: Metabolic and hemodynamic responses at rest, during and after exercise in T2D patients and control subjects during placebo and CoQ10 conditions.

\begin{tabular}{|c|c|c|c|c|c|c|c|}
\hline \multirow{2}{*}{\begin{tabular}{|l|} 
Group \\
Trial \\
\end{tabular}} & \multicolumn{2}{|l|}{ T2D (n = 7) } & \multicolumn{2}{|c|}{ Control (n= 7) } & \multirow{2}{*}{\begin{tabular}{|l}
$P$ \\
Group
\end{tabular}} & \multirow{2}{*}{$\begin{array}{l}P \\
\text { Tx }\end{array}$} & \multirow{2}{*}{$\begin{array}{l}P \\
\text { Inter }\end{array}$} \\
\hline & Placebo & $\mathrm{CoQ}_{10}$ & Placebo & $\mathrm{CoQ}_{10}$ & & & \\
\hline \multicolumn{8}{|l|}{ Resting } \\
\hline $\mathrm{PCr}(\mathrm{mM})$ & $39.12 \pm 1.98$ & $38.93 \pm 3.66$ & $41.09 \pm 2.13$ & $39.82 \pm 1.76$ & 0.459 & 0.637 & 0.358 \\
\hline $\mathrm{Pi}(\mathrm{mM})$ & $5.84 \pm 1.07$ & $4.99 \pm 1.75$ & $4.78 \pm 1.06$ & $4.61 \pm 0.95$ & 0.237 & 0.518 & 0.695 \\
\hline $\mathrm{ipH}$ & $7.02 \pm 0.02$ & $7.00 \pm 0.02$ & $7.01 \pm 0.04$ & $7.02 \pm 0.01$ & 0.329 & 0.319 & 0.218 \\
\hline \multicolumn{8}{|l|}{ Exercise } \\
\hline SSI Ex. Duration (s) & $312.8 \pm 117.6$ & $321.7 \pm 135.5$ & $408.9 \pm 103.5$ & $424.6 \pm 88.3$ & 0.030 & 0.720 & 1.00 \\
\hline \multicolumn{8}{|l|}{ NIRS } \\
\hline $\mathrm{T}_{50}$ & $70.93 \pm 52.13$ & $69.10 \pm 33.72$ & $31.83 \pm 8.36$ & $32.43 \pm 10.02$ & 0.004 & 0.960 & 0.920 \\
\hline BFindex $(\% / s)$ & $5.33 \pm 2.88$ & $3.99 \pm 2.23$ & $9.74 \pm 2.92$ & $9.22 \pm 2.42$ & 0.010 & 0.469 & 0.736 \\
\hline \multicolumn{8}{|l|}{ MRS } \\
\hline End PCr $(\mathrm{mM})$ & $45.58 \pm 17.24$ & $42.13 \pm 4.68$ & $49.4 \pm 17.60$ & $51.76 \pm 14.57$ & 0.562 & 0.632 & 0.980 \\
\hline $\mathrm{PCr}_{\mathrm{re}}\left(\mathrm{mM} \cdot \mathrm{kg} \cdot \mathrm{min}^{-1}\right)$ & $22.31 \pm 13.35$ & $23.20 \pm 11.39$ & $49.37 \pm 36.61$ & $43.74 \pm 33.28$ & 0.034 & 0.905 & 0.960 \\
\hline Tc (s) & $67.21 \pm 69.51$ & $53.61 \pm 22.55$ & $35.59 \pm 23.65$ & $32.54 \pm 15.18$ & 0.014 & 0.802 & 0.770 \\
\hline pH End ex. (12-s) & $6.979 \pm 0.074$ & $6.96 \pm 0.036$ & $7.002 \pm 0.036$ & $7.004 \pm 0.049$ & 0.094 & 0.663 & 0.586 \\
\hline pH 1 min post ex. (12-s) & $6.886 \pm 0.064$ & $6.915 \pm 0.063$ & $6.972 \pm 0.038$ & $6.988 \pm 0.059$ & 0.001 & 0.311 & 0.793 \\
\hline
\end{tabular}

Note: PCr: Phosphocreatine; Pi: Inorganic phosphate; ipH: intracellular $\mathrm{pH} ; \mathrm{T}_{50 \mathrm{NIR}}$ : Time to $50 \%$ of Hemoglobin/Myoglobinreoxygenation from cessation of exercise; BFindex: Blood flow index; End PCr: End exercise $\mathrm{PCr}$; $\mathrm{PCr}_{\mathrm{re}}$ : $\mathrm{Phosphocreatine}$ resynthesis rate; Tc: Time constant; 12-s: 12-s all out exercise; SSI: steady state incremental exercise. Data are expressed in mean \pm SD.

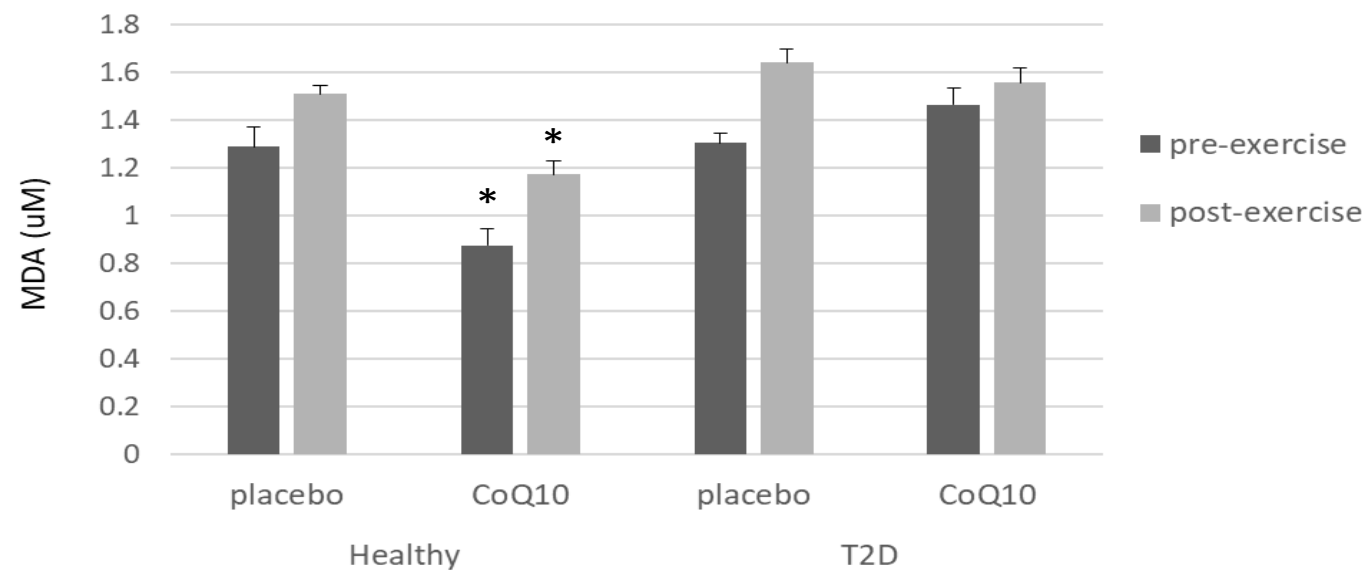

Figure 4: Changes in MDA (uM) during placebo and $\mathrm{CoQ}_{10}$ trials at pre-exercise (blue bars) and post-exercise (red bars) states in control subjects (left panel) and T2D patients (right panel).

"Significantly different from placebo condition at $p<0.05$.

(Figure 4). However, improvements in exercise duration after $\mathrm{CoQ}_{10}$ supplementation was not statistically different for either group (Table 2). Further, $\mathrm{PCr}$ changes at the onset of exercise, end exercise ipH, or $\triangle \mathrm{BV}$ during $\mathrm{SSI}$ exercise was not significantly altered for either group with $\mathrm{CoQ}_{10}$.

Recovery response: $\mathrm{PCr}_{\mathrm{re}}$ after 12-s exercise, was not significantly different for either group under $\mathrm{CoQ}_{10}$ trial (Table 2 and Figure 5). Slight improvement in ipH recovery was seen in T2D after CoQ10 trial (6.89 vs. 6.92). However, these changes were not statistically significant $(p>0.05)$. $\mathrm{Hb} / \mathrm{Mb}$-oxy during recovery was not significantly different with $\mathrm{CoQ}_{10}$ treatment for ei- ther T2D or control group ( $p=0.960$ ) (Table 2 and Figure 5). Moreover, no significant $\mathrm{CoO}_{10}$ treatment effect $(p=$ 0.469) was detected in BFindex for either group.

\section{Discussion}

\section{T2D Patients vs. Control subjects}

Resting response: This study found a significantly lower $\mathrm{PCr}, \mathrm{PCr} / \mathrm{Pi}$ and higher $\mathrm{Pi}$ in T2D when compared to control subjects. Studies have reported abnormal resting $\mathrm{Pi}$ metabolites and/or ratios in different diseases. For example, lower $\mathrm{PCr} / \mathrm{Pi}$ has been reported in mitochondrial [29] and COPD patients [30], while higher $\mathrm{Pi} / \mathrm{PCr}$ has been associated with $\mathrm{T} 2 \mathrm{D}$ 


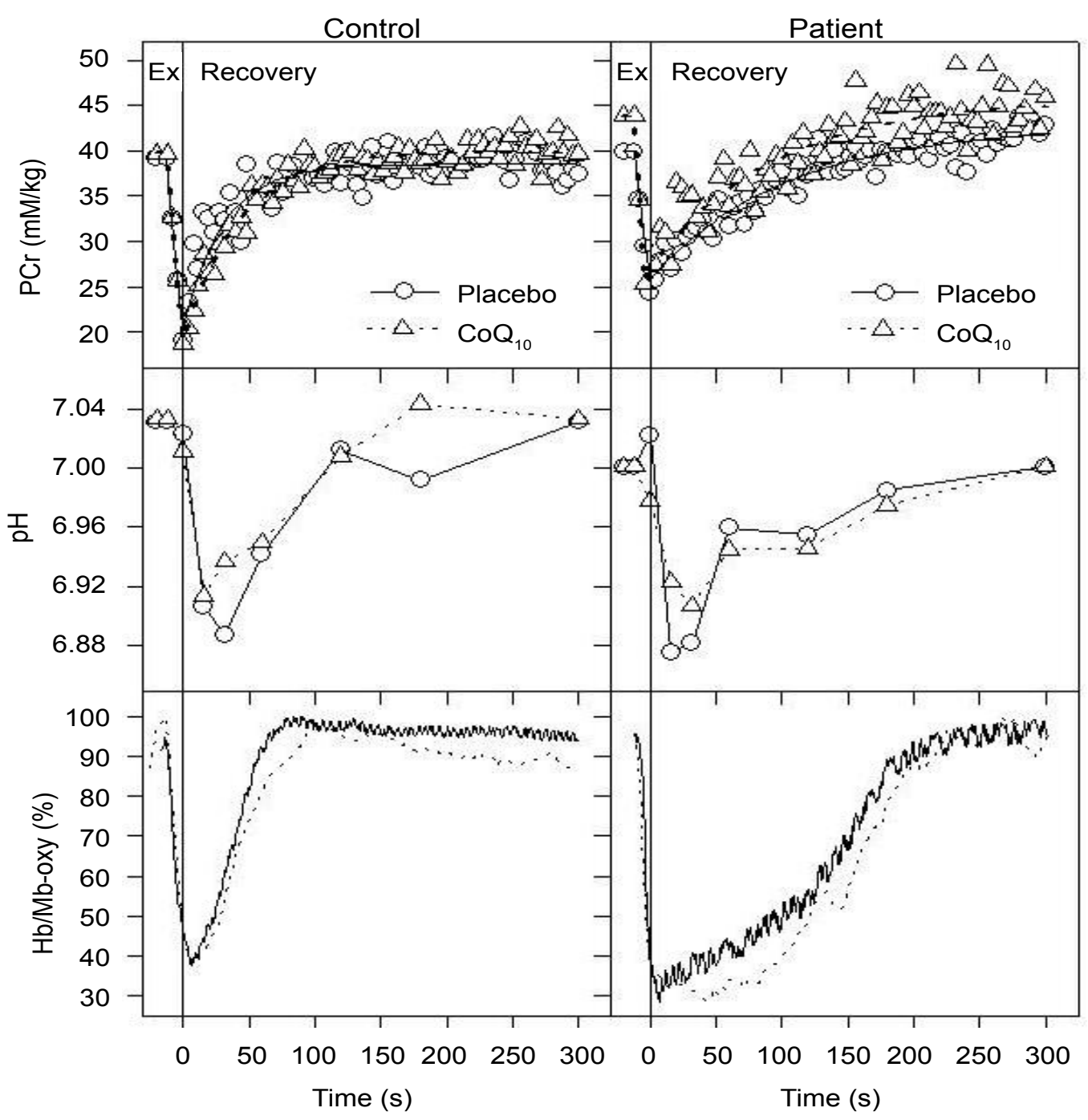

Figure 5: Changes in PCr (top panel), ipH (middle panel), Hb/Mb-oxy (bottom panel) during and after 12-s exercise in control subjects (left panels) and T2D patients (right panels) during placebo (circle and solid line) and $\mathrm{CoQ}_{10}$ trials (triangle, dotted line).

[31,32]. Under the condition of constant $\mathrm{pH}, \mathrm{PCr} / \mathrm{Pi}$ is interpreted as phosphorylation potential, one of the main activators in cellular respiration [33,34]. Thus, lower resting $\mathrm{PCr} / \mathrm{Pi}$ observed in T2D suggests a state of constant metabolic stress even at rest and a reduced phosphorylation potential. Under ischemic conditions, this reduced phosphorylation potential results not only in over delivered electrons $\left(e^{-}\right)$and subsequent over production of ROS but also accumulation of other metabolites, such as $\mathrm{H}^{+}, \mathrm{Pi}$, and ADP, which negatively impact cellular respiration and further stress the mitochondrial respiratory system.

Exercise and recovery responses: One of the research questions in this study was whether T2D patients have impaired skeletal muscle blood flow, mitochondrial function and exercise performance when compared to control subjects. To compare groups, this study used the same relative workloads for all subjects to keep the metabolic rate equivalent. During this state, $\mathrm{PCr}_{\text {re }}$ was significantly less in T2D suggesting compromised mitochondrial oxidative phosphorylation capacity. Delayed $\mathrm{PCr}_{\text {re }}$ can occur when mitochondria is not functioning properly. Delayed $\mathrm{PCr}_{\mathrm{re}}$ can also occur when there is an insufficient $\mathrm{O}_{2}$ supply to the mitochondria as evidenced by a significant relationship between $\mathrm{O}_{2}$ recovery and $\mathrm{PCr}$ recovery $(p<0.01)$ seen in this study. These observations are in agreement with other studies reporting delayed $\mathrm{PCr}$ recovery after exercise under ischemic condition $[35,36]$. Insufficient $\mathrm{O}_{2}$ supply to the mitochondria impairs e- flow in the ETC and, as a result, ATP production is reduced. Furthermore, insufficient $\mathrm{O}_{2}$ delivery while the mitochondria is fully activated during exercise can lead to overflow of $\mathrm{e}^{-}$within the mitochondria increasing redox potential that favors ROS production.

In this study, a reduction in capillary blood flow (as indicated by lower $\triangle B V$ and BFindex) was observed in T2D during exercise. This is in agreement with other studies reporting reduced skeletal muscle blood flow in response to exercise in T2D $[37,38]$. A lower $\triangle B V$ and BFindex found in T2D indicates reduced $\mathrm{O}_{2}$ supply to the muscle. When there is insufficient $\mathrm{O}_{2}$ supply, while the demand is high, a mismatch in the $\mathrm{O}_{2}$ flux can occur, impairing mitochondrial oxidative phosphorylation capacity. This phenomenon was clearly demonstrated where 
$\mathrm{PCr}$ recovery was delayed in $\mathrm{T} 2 \mathrm{D}$ and it was significantly related to $\mathrm{T}_{50 \mathrm{NIR}}$ and $\triangle \mathrm{BV}$.

Intracellular pH response: Exercise capacity of T2D was significantly lower than control subjects where T2D patients reached $\mathrm{PCr} / \mathrm{Pi}$ of 1 (a state of anaerobic threshold), earlier than control subjects. Moreover, T2D patients' ipH during exercise was lower and recovery was significantly delayed in T2D (Figure 4 and Figure 5). The extent of $\mathrm{H}^{+}$generation depends on work intensity and ATP hydrolysis via anaerobic glycolysis. Moreover, the amount of $\mathrm{H}^{+}$present in the cell depends on the ability of $\mathrm{H}^{+}$efflux. In this study, exercise was stopped at the same metabolic state (anaerobic threshold) for all subjects. Therefore, lower intramuscular $\mathrm{pH}$ and delayed $\mathrm{pH}$ recovery in $\mathrm{T} 2 \mathrm{D}$ reflects the reduced ability of $\mathrm{H}^{+}$to be released from the cytosol to the extracellular space rather than increased ATP hydrolysis. Impaired blood flow $\left(\mathrm{O}_{2}\right.$ delivery) observed in T2D appears to be the primary factor for the reduced clearance capacity of $\mathrm{H}^{+}$. It has been suggested that the relationship between exercise-induced $\mathrm{PCr} / \mathrm{Pi}$ and acidosis is assumed to be a specific parameter for muscle ischemia [39,40]. At similar $\mathrm{PCr} / \mathrm{Pi}$, increased acidosis suggests delayed clearance of lactate or $\mathrm{H}^{+}$due to reduced tissue perfusion as evidenced by reduced $\triangle B V$ and BFindex. Furthermore, ipH kinetics can affect $\mathrm{PCr}$ recovery. Thus delayed $\mathrm{PCr}$ recovery, associated with reduced $\mathrm{O}_{2}$ delivery, can be further delayed by prolonged acidic environment.

\section{Effect of $\mathrm{CoQ}_{10}$}

Resting response: After $\mathrm{CoQ}_{10}$ trial, a significant reduction in MDA was observed in healthy, older control subjects, suggesting $\mathrm{CQ}_{10}$ may have beneficial antioxidant effects in reducing oxidative stress. This is in agreement with other studies, which have reported a positive antioxidant role of $\mathrm{CoQ}_{10}$ lowering MDA level [23,41-43]. One study reported reduction of MDA with $\mathrm{CoQ}_{10}$ supplementation in children with autism spectrum disorder [42]. Another study reported similar findings in patients with multiple sclerosis [43]. Aging is often associated with elevated oxidative stress [44], causing age related tissue damage. Furthermore, reduced level of CoQ has been shown as a result of the aging process [45], making the older individuals even more prone to oxidative damage. Thus, supplementing with $\mathrm{CoQ}_{10}$ can potentially benefit this population by normalizing $\mathrm{CoQ}_{10}$ and also scavenging ROS, thereby reducing oxidative stress and delaying the aging process.

Interestingly, MDA level was not significantly altered with $\mathrm{CoQ}_{10}$ in T2D patient group. Although studies have suggested the beneficial antioxidant effect of $\mathrm{CoQ}_{10}$, clinical trials involving particularly at high-risk patients, such as T2D, are less remarkable [46]. Lack of significant changes seen in T2D patients in this study may partly be due to the dosage or the duration not potent or long enough for this population, who may have undergone significant cellular modifications with chronic exposure to oxidative stress. For example, damaged mitochondria of type II diabetes can lead to not only deficient in CoQ $[20,21]$ but also over production of $\mathrm{e}^{-}$and subsequent ROS, creating a greater oxidative stress environment than healthy, older control subjects. The given dose or duration of $\mathrm{CoQ}_{10}$ in current study may have not been sufficient enough to scavenge the over delivered $\mathrm{e}^{-}$and/ or reduce ROS present within the T2D mitochondria, which may be responsible for the lack of reduction in MDA level seen in this population.

Metabolic response: After the $\mathrm{CoQ}_{10}$ treatment, T2D group's PCr and PCr/Pi were elevated to the values similar to the control subjects. However, these differences were not significant. Normalization of $\mathrm{PCr} / \mathrm{Pi}$ ratio with $\mathrm{CoQ}_{10}$ has been seen in patients with mitochondrial disease [47] and Friedreich's Ataxia [48] $\mathrm{CoQ}_{10}$ can improve the metabolic status by better handling of the $\mathrm{e}^{-}$within ETC, where $\mathrm{CoQ}_{10}$ can scavenge over delivered $\mathrm{e}^{-}$reducing ROS concentration and also enhancing $e^{-}$transport as a co-factor to improve ATP production [22,49].

Exercise and recovery responses: Similar to the resting response, a significant reduction in MDA, with $\mathrm{CoQ}_{10}$ supplementation, was observed in control subjects although improvements in functional capacity or other measures were not as obvious. This is contrary to our original hypothesis that $\mathrm{CoQ}_{10}$ would improve functional capacity of T2D patients. Lack of improvements seen in this study may be due to multiple factors. First and foremost, two weeks of $\mathrm{CoQ}_{10}$ supplementation at $200 \mathrm{mg} \cdot \mathrm{day}^{-1}$ may have not been long enough or potent enough for those already having undergone significant modification of vascular tone with chronic exposure to oxidative stress. For the study, a short term but a maximum dose at safe level of $200 \mathrm{mg} \cdot \mathrm{day}^{-1}$ (typical dosage $100 \mathrm{mg} \cdot \mathrm{day}^{-1}$ ) was chosen to see the acute effect of the supplementation. Although some studies have reported positive effects of $\mathrm{CoQ}_{10}$ supplementation with a similar duration and dosage $[50,51]$, results from $\operatorname{CoQ}_{10}$ studies involving human subjects, particularly patient populations, are rather inconclusive [52]. Solid evidence for the benefit of short-term $\operatorname{CoQ}_{10}$ supplementation on T2D with PAD has yet to be established.

Another possible factor may be due to $\mathrm{CoQ}_{10}$ having a greater effect on the mitochondria than microvasculature. As described earlier, $\mathrm{PCr}_{\text {re }}$ can be altered significantly by $\mathrm{O}_{2}$ delivery as evidenced by current findings of strong and significant relationships between $\mathrm{PCr}_{\text {re }}$ and hemodynamic measures. This phenomenon is in agreement with other studies reporting the influence of $\mathrm{O}_{2}$ delivery on mitochondrial phosphorylation capacity. Thus, while some improvements in mitochondrial oxidative phosphorylation capacity may have been pre- 
sent, without sufficient $\mathrm{O}_{2}$ delivery in mitochondria, no discernable improvements in $\mathrm{PCr}_{\text {re }}$ can be observed.

\section{Limitations}

Small sample size of the patient group as well as the control group decreases the statistical power, which makes it challenging to detect the small changes and draw a generalization of the population.

\section{Conclusion}

In conclusion, $\mathrm{CoO}_{10}$ supplementation exhibited a positive effect in lowering oxidative stress status, shown by reduced MDA, in healthy older individuals. Moreover, $\mathrm{CoQ}_{10}$ supplementation appeared to have marginal effect on mitochondrial oxidative phosphorylation potential of T2D patients at rest without significantly affecting microvascular measures or functional capacity during exercise. Lack of significant microvascular changes after two weeks of $\mathrm{CoQ}_{10}$ supplementation in T2D is most likely the reason for the lack of functional improvement in T2D.

\section{Acknowledgement}

We dedicate this paper to late scientist, Britton Chance, who had been the inspiration and tremendous support for this work. We would also like to thank Ms. Gwen Lech, Dr. Emile R. Mohler III, Allen Bonner, Dr. Luis Melara and Dr. Paul Taylor for their assistance and support.

The authors declare that the research was conducted in the absence of any commercial or financial relationships that could be construed as a potential conflict of interest.

\section{Source of Support}

This work was supported by Diabetes Action Research and Education Foundation Grant.

\section{Statement of Equal Authors' Contribution}

All authors have read the manuscript, assisted in all aspects of the research study as well as the writing of the manuscript, and have agreed to the publication of this manuscript.

\section{References}

1. Centers for Disease Control and Prevention (2014) National diabetes statistics report: Estimates of diabetes and its burden in the United States, 2014. Department of Health and Human Services, Atlanta, GA, US.

2. Mapanga RF, Essop MF (2016) Damaging effects of hyperglycemia on cardiovascular function: Spotlight on glucose metabolic pathways. Am J Physiol Heart Circ Physiol 310: H153-H173.

3. Guzik TJ, Mussa S, Gastaldi D, Sadowski J, Ratnatunga C, et al. (2002) Mechanisms of increased vascular superoxide production in human diabetes mellitus: Role of $N A D(P) H$ oxidase and endothelial nitric oxide synthase. Circulation 105: 1656-1662.
4. Evans JL, Maddux BA, Goldfine ID (2005) The molecular basis for oxidative stress-induced insulin resistance. Antioxidants Redox Signal 7: 1040-1052.

5. Kjaer LK, Oellgaard J, Henriksen T, Gaede P, Pedersen $\mathrm{O}$, et al. (2018) Indicator of RNA oxidation in urine for the prediction of mortality in patients with type 2 diabetes and microalbuminuria: A post-hoc analysis of the Steno-2 trial. Free Radic Biol Med 129: 247-255.

6. Brownlee M (2001) Biochemistry and molecular cell biology of diabetic complications. Nature 414: 813-820.

7. Park K, Gross M, Lee DH, Holvoet $P$, Himes $\mathrm{JH}$, et al. (2009) Oxidative stress and insulin resistance: The coronary artery risk development in young adults study. Diabetes Care 32: 1302-1307.

8. Odegaard AO, Jacobs DR, Sanchez OA, Goff DC, Reiner AP, et al. (2016) Oxidative stress, inflammation, endothelial dysfunction and incidence of type 2 diabetes. Cardiovasc Diabetol 15: 51.

9. Marso SP, Hiatt WR (2006) Peripheral arterial disease in patients with diabetes. J Am Coll Cardiol 47: 921-929.

10. Grenon SM, Chong K, Alley H, Nosova E, Gasper W, et al. (2014) Walking disability in patients with peripheral artery disease is associated with arterial endothelial function. $J$ Vasc Surg 59: 1025-1034.

11. Gardner AW, Parker DE, Montgomery PS, Sosnowska D, Casanegra Al, et al. (2015) Influence of diabetes on ambulation and inflammation in men and women with symptomatic pheripheral artery disease. J Clin Transl Endocrinol 2: $137-143$.

12. Vrsalovic M, Vucur K, Vrsalovic Presecki A, Fabijanic D, Milosevic M, et al. (2017) Impact of diabetes on mortality in peripheral artery disease: A meta-analysis. Clin Cardiol 40: $287-291$

13. Liu Y, Mei X, Li J, Lai N, Yu X (2016) Mitochondrial function assessed by 31P MRS and BOLD MRI in non-obese type 2 diabetic rats. Physiol Rep 4: e12890.

14. Pottecher J, Adamopoulos C, Lejay A, Bouitbir J, Charles $\mathrm{AL}$, et al. (2018) Diabetes worsens skeletal muscle mitochondrial function, oxidative stress, and apoptosis after lower-limb ischemia-reperfusion: Implication of the RISK and SAFE Pathways? Front Physiol 9: 579.

15. Fetterman JL, Holbrook M, Westbrook DG, Brown JA, Feeley KP, et al. (2016) Mitochondrial DNA damage and vascular function in patients with diabetes mellitus and atherosclerotic cardiovascular disease. Cardiovasc Diabetol 15: 53.

16. Beckman JA, Creager MA, Libby $P$ (2002) Diabetes and atherosclerosis: Epidemiology, pathophysiology, and management. JAMA 287: 2570-2581.

17. Raminderjit K, Manpreet K, Jatinder S (2018) Endothelial dysfunction and platelet hyperactivity in type 2 diabetes mellitus: Molecular insights and therapeutic strategies. Cardiovasc Diabetol 17: 121.

18. Chung SS, Ho EC, Lam KS, Chung SK (2003) Contribution of polyol pathway to diabetes-induced oxidative stress. J Am Soc Nephrol 14: S233-S236.

19. Ziegler D, Sohr CG, Nourooz-Zadeh J (2004) Oxidative stress and antioxidant defense in relation to the severity of diabetic polyneuropathy and cardiovascular autonomic neuropathy. Diabetes Care 27: 2178-2183.

20. Hasegawa G, Yamamoto Y, Zhi JG, Tanino Y, Yamasaki $\mathrm{M}$, et al. (2005) Daily profile of plasma \%CoQ10 level, a 
biomarker of oxidative stress, in patients with diabetes manifesting postprandial hyperglycaemia. Acta Diabetol 42: 179-181.

21. Ates O, Bilen H, Keles S, Alp HH, Keleş MS, et al. (2013) Plasma coenzyme Q10 levels in type 2 diabetic patients with retinopathy. Int J Ophthalmol 6: 675-679.

22. Raygan F, Rezavandi Z, Dadkhah T, Farrokhian A, Asemi Z (2016) The effects of coenzyme Q10 administration on glucose homeostasis parameters, lipid profiles, biomarkers of inflammation and oxidative stress in patients with metabolic syndrome. Eur J Nutr 55: 2357-2364.

23. Abdollahzad H, Aghdashi M, Asghari J, Alipour B (2015) Effects of coenzyme Q10 supplementation on inflammatory cytokines (TNF-a, IL-6) and oxidative stress in rheumatoid arthritis patients: A randomized controlled trial. Arch Med Res 46: 527-533.

24. Jiménez-Santos MA, Juárez-Rojop IE, Tovilla-Zárate CA Espinosa-García MT, Juárez-Oropeza MA, et al. (2014) Coenzyme Q10 supplementation improves metabolic parameters, liver function and mitochondrial respiration in rats with high doses of atorvastatin and a cholesterol-rich diet. Lipids Health Dis 13: 22.

25. Cornelius N, Wardman JH, Hargreaves IP, Neergheen $\mathrm{V}$, Bie AS, et al. (2017) Evidence of oxidative stress and mitochondrial dysfunction in spinocerebellar ataxia type 2 (SCA2) patient fibroblasts: Effect of coenzyme Q10 supplementation on these parameters. Mitochondrion 34: 103114.

26. Gao L, Mao Q, Cao J, Wang Y, Zhou X, et al. (2012) Effects of coenzyme Q10 on vascular endothelial function in humans: A meta-analysis of randomized controlled trials. Atherosclerosis 221: 311-316.

27. Cicero AF, Morbini M, Rosticci M, D"Addato S, Grand E, et al. (2016) Middle-term dietary supplementation with red yeast rice plus coenzyme Q10 improves lipid pattern, endothelial reactivity and arterial stiffness in moderately hypercholesterolemic Subjects. Ann Nutr Metab 68: 213219.

28. Walter G, Vandenborne K, Mc Cully KK, Scott Leigh J (1997) Noninvasive measurement of phosphocreatine recovery kinetics in single human muscles. Am J Physiol 272: 525-534.

29. Nachbauer W, Boesch S, Schneider R, Eigentler A, Wanschitz J, et al. (2013) Bioenergetics of the calf muscle in Friedreich ataxia patients measured by 31P-MRS before and after treatment with recombinant human erythropoietin. PLoS One 8: e69229.

30. Layec G, Hart CR, Trinity JD, Kwon OS, Rossman MJ, et al (2017) Oxygen delivery and the restoration of the muscle energetic balance following exercise: Implications for delayed muscle recovery in patients with COPD. Am J Physiol Endocrinol Metab 313: E94-E104.

31. Greenman RL, Panasyuk S, Wang X, Lyons TE, Dinh T, et al. (2005) Early changes in the skin microcirculation and muscle metabolism of the diabetic foot. Lancet 366: 17111717.

32. Ripley EM, Clarke GD, Hamidi V, Martinez RA, Settles FD, et al. (2018) Reduced skeletal muscle phosphocreatine concentration in type 2 diabetic patients: A quantitative image-based phosphorus-31 MR spectroscopy study. Am J Physiol Endocrinol Metab 315: E229-E239.

33. Taylor DJ, Krige D, Barnes PR, Kemp GJ, Carroll MT, et al. (1994) A 31P magnetic resonance spectroscopy study of mitochondrial function in skeletal muscle of patients with Parkinson's disease. J Neurol Sci 125: 77-81.

34. Chance B, Eleff S, Leigh JS, Sokolow D, Sapega A (1981) Mitochondrial regulation of phosphocreatine/inorganic phosphate ratios in exercising human muscle: $A$ gated $31 \mathrm{P}$ NMR study. Proc Natl Acad Sci U S A 78: 6714-6718.

35. Esterhammer R, Schocke M, Gorny O, Posch L, Messner $\mathrm{H}$, et al. (2008) Phosphocreatine kinetics in the calf muscle of patients with bilateral symptomatic peripheral arterial disease during exhaustive incremental exercise. Mol Imaging Biol 10: 30-39.

36. Sedivy P, Drobny M, Dezortova M, Herynek V, Roztocil K, et al. (2018) 31P-MR spectroscopy in patients with mild and serious lower limb ischemia. Int Angiol 37: 293-299.

37. Kingwell BA, Formosa M, Muhlmann M, Bradley S, Conell G, et al. (2003) Type 2 diabetic individuals have impaired leg blood flow responses to exercise: Role of endothelium-dependent vasodilation. Diabetes Care 26: 899-904.

38. Womack L, Peters D, Barrett EJ, Kaul S, Price W, et al. (2009) Abnormal skeletal muscle capillary recruitment during exercise in patients with type 2 diabetes mellitus and microvascular complications. J Am Coll Cardiol 53: 21752183.

39. Schunk K, Romaneehsen B, Rieker O, Düber C, Kersjes W, et al. (1998) Dynamic phosphorus-31 magnetic resonance spectroscopy in arterial occlusive disease: Effects of vascular therapy on spectroscopic results. Invest Radiol 33: 329-335.

40. Martin A, Albrecht IS, Mohammad K, Daniel D, Bartko J, et al. (2011) Effect of ischemic preconditioning in skeletal muscle measured by functional magnetic resonance imaging and spectroscopy: A randomized crossover trial. J Cardiovasc Magn Reson 13: 32.

41. Gholami M, Zarei P, Sadeghi SB, Rafiei F, Khosrowbeygi A (2018) Effects of coenzyme Q10 supplementation on serum values of adiponectin, leptin, 8-isoprostane and malondialdehyde in women with type 2 diabetes. Gynecol Endocrinol 34: 1059-1063.

42. Mousavinejad E, Ghaffari MA, Riahi F, Hajmohammadi M, Tiznobeyk Z, et al. (2018) Coenzyme Q10 supplementation reduces oxidative stress and decreases antioxidant enzyme activity in children with autism spectrum disorders. Psychiatry Res 265: 62-69.

43. Sanoobar M, Eghtesadi S, Azimi A, Khalili M, Jazayeri S, et al. (2003) Coenzyme Q10 supplementation reduces oxidative stress and increases antioxidant enzyme activity in patients with relapsing-remitting multiple sclerosis. Int $\mathrm{J}$ Neurosci 123: 776-782.

44. Battino M, Gorini A, Villa RF, Genova ML, Bovina C, et al. (1995) Coenzyme $Q$ content in synaptic and non-synaptic mitochondria from different brain regions in the ageing rat. Mech Ageing Dev 78: 173-187.

45. Finkel T, Holbrook NJ (2000) Oxidants, oxidative stress and the biology of ageing. Nature 408: 239-247.

46. Suksomboon N, Poolsup N, Juanak N (2015) Effects of coenzyme Q10 supplementation on metabolic profile in diabetes: A systematic review and meta-analysis. J Clin Pharm Ther 40: 413-418.

47. Barbiroli B, lotti S, Lodi R (1999) Improved brain and muscle mitochondrial respiration with $\mathrm{CoQ}$ : An in vivo study by 31P-MR spectroscopy in patients with mitochondrial cytopathies. Biofactors 9: 253-260. 
48. Lodi R, Hart PE, Rajagopalan B, Taylor DJ, Crilley JG, et al (2001) Antioxidant treatment improves in vivo cardiac and skeletal muscle bioenergetics in patients with Friedreich's ataxia. Ann Neurol 49: 590-596.

49. Acosta MJ, Vazquez Fonseca L, Desbats MA, Cerqua C, Zordan R, et al. (2016) Coenzyme Q biosynthesis in health and disease. Biochim Biophys Acta 1857: 1079-1085.

50. Raitakari OT, McCredie RJ, Witting P, Griffiths KA, Letters $J$, et al. (2000) Coenzyme Q improves LDL resistance to ex vivo oxidation but does not enhance endothelial function in hypercholesterolemic young adults. Free Radic Biol Med 28: 1100-1105.

51. Emami A, Bazargani-Gilani B (2018) Effect of oral CoQ10 supplementation along with precooling strategy on cellular response to oxidative stress in elite swimmers. Food Funct 9: 4384-4393.

52. Hernández-Camacho JD, Bernier M, López-Lluch G, Navas $P(2018)$ Coenzyme Q10 supplementation in aging and disease. Front Physiol 9: 44. 